\title{
Socioeconomic inequalities in hospitalizations for chronic ambulatory care sensitive conditions: a systematic review of peer-reviewed literature, 1990-2018
}

\author{
Lauren E. Wallar, Eric De Prophetis and Laura C. Rosella*
}

\begin{abstract}
Background: Hospitalizations for chronic ambulatory care sensitive conditions are an important indicator of health system equity and performance. Chronic ambulatory care sensitive conditions refer to chronic diseases that can be managed in primary care settings, including angina, asthma, and diabetes, with hospitalizations for these conditions considered potentially avoidable with adequate primary care interventions. Socioeconomic inequities in the risk of hospitalization have been observed in several health systems globally. While there are multiple studies examining the association between socioeconomic status and hospitalizations for chronic ambulatory care sensitive conditions, these studies have not been systematically reviewed. The objective of this study is to systematically identify and describe socioeconomic inequalities in hospitalizations for chronic ambulatory care sensitive conditions amongst adult populations in economically developed countries reported in high-quality observational studies published in the peer-reviewed literature.

Methods: Peer-reviewed literature was searched in six health and social science databases: MEDLINE, EMBASE, PsycInfo, CINAHL, ASSIA, and IBSS using search terms for hospitalization, socioeconomic status, and chronic ambulatory care sensitive conditions. Study titles and abstracts were first screened followed by full-text review according to the following eligibility criteria: 1) Study outcome is hospitalization for selected chronic ambulatory care sensitive conditions; 2) Primary exposure is individual- or area-level socioeconomic status; 3) Study population has a mean age $\pm 1 \mathrm{SD}<75$ years of age; 4) Study setting is economically developed countries; and 5) Study type is observational. Relevant data was then extracted, and studies were critically appraised using appropriate tools from The Joanna Briggs Institute. Results were narratively synthesized according to socioeconomic constructs and type of adjustment (minimally versus fully adjusted).

Results: Of the 15,857 unique peer-reviewed studies identified, 31 studies met the eligibility criteria and were of sufficient quality for inclusion. Socioeconomic constructs and hospitalization outcomes varied across studies. However, despite this heterogeneity, a robust and consistent association between lower levels of socioeconomic status and higher risk of hospitalizations for chronic ambulatory care sensitive conditions was observed.

(Continued on next page)
\end{abstract}

\footnotetext{
*Correspondence: laura.rosella@utoronto.ca

Dalla Lana School of Public Health, University of Toronto, 155 College St,

Toronto, ON M5T 3M7, Canada
}

(c) The Author(s). 2020 Open Access This article is licensed under a Creative Commons Attribution 4.0 International License, which permits use, sharing, adaptation, distribution and reproduction in any medium or format, as long as you give appropriate credit to the original author(s) and the source, provide a link to the Creative Commons licence, and indicate if changes were made. The images or other third party material in this article are included in the article's Creative Commons licence, unless indicated otherwise in a credit line to the material. If material is not included in the article's Creative Commons licence and your intended use is not permitted by statutory regulation or exceeds the permitted use, you will need to obtain permission directly from the copyright holder. To view a copy of this licence, visit http://creativecommons.org/licenses/by/4.0/ The Creative Commons Public Domain Dedication waiver (http://creativecommons.org/publicdomain/zero/1.0/) applies to the data made available in this article, unless otherwise stated in a credit line to the data. 


\begin{abstract}
(Continued from previous page)
Conclusions: This systematic review is the first to comprehensively identify and analyze literature on the relationship between SES and hospitalizations for chronic ambulatory care sensitive conditions, considering both aggregate and condition-specific outcomes that are common to several international health systems. The evidence consistently demonstrates that lower socioeconomic status is a risk factor for hospitalization across global settings. Effective health and social interventions are needed to reduce these inequities and ensure fair and adequate care across socioeconomic groups.
\end{abstract}

Trial registration: PROSPERO CRD42018088727.

Keywords: Ambulatory care sensitive conditions, Hospitalization, Socioeconomic status, Health inequalities, Observational studies, Systematic review

\section{Background}

Socioeconomic inequalities in hospitalizations for ambulatory-care sensitive conditions (ACSCs), also known as avoidable or preventable hospitalizations, have been observed in multiple countries including Canada [1], the United States [2], England [3], France [4], Portugal [5], Australia [6], and others, where individuals of lower socioeconomic status (SES) are at higher risk of hospitalization compared to higher SES individuals. While the number and type of disease conditions considered sensitive to ambulatory or primary care differ [7], the underlying concept of ACSC hospitalizations is similar across health systems. ACSC hospitalizations refer to hospitalizations for certain disease conditions that are thought to be avoided if patients had received timely and effective primary care to prevent disease or disease exacerbations [8]. Hospitalization rates are routinely monitored by health systems as an indicator of health system performance at the nexus of primary and acute care $[9,10]$. Socioeconomic inequalities in ACSC hospitalization rates may additionally indicate health system inequities along the care continuum that differentially affects the effectiveness of preventive and primary care received across socioeconomic groups [11] and may signal access barriers within a health system or other structural determinants of health. Thus, monitoring socioeconomic inequalities and understanding their root causes is important to improving health system performance and decreasing unnecessary hospitalizations.

SES is one of the most studied risk factors for ACSC hospitalizations. First described by Billings et al. in New York City [8], multiple studies have independently observed an association between SES and ACSC hospitalizations [12-16]. However, to our knowledge, these studies have not been systematically described. Previous narrative and systematic reviews of ACSC hospitalizations have focused on other risk factors including race and ethnicity $[17,18]$, primary care organization $[19,20]$, and other potential causes of geographic variation [21]. While the relationship between SES and hospitalizations for specific ACSCs such as asthma [22], congestive heart failure
(CHF) [23], and chronic obstructive pulmonary disease (COPD) [24] have previously been reviewed, a comprehensive review considering the effect of SES on hospitalizations for ACSCs in aggregate and by condition has not been conducted. One particular challenge for this type of review is heterogeneity in the definitions of SES and ACSC hospitalizations [7]. To address this, this review adopts a more specific definition of SES and focuses only on chronic ACSCs that are consistent with the Canadian definition, namely angina, asthma, CHF, COPD, diabetes, epilepsy, and hypertension [9]. These conditions are common to other international health system definitions $[10,25,26]$.

The objective of this review is to systematically identify and describe socioeconomic inequalities in chronic ACSC hospitalizations amongst adult populations in economically developed countries reported in high-quality observational studies published in the peer-reviewed literature.

\section{Methods \\ Searches}

MEDLINE, EMBASE, PsycInfo, CINAHL (Cumulative Index to Nursing and Allied Health Literature), ASSIA (Applied Social Sciences Index and Abstracts), and IBSS (International Bibliography of the Social Sciences) databases were searched from January 1, 1990 - July 31, 2018. Search strategies were iteratively developed and organized into three main blocks of terms by selecting and combining appropriate $\mathrm{MeSH}$ and keyword terms for 1) hospitalizations, 2) SES, and 3) ACSCs. Hospitalization terms were identified using the $\mathrm{MeSH}$ browser for relevant and related terms. SES terms were identified by consulting search strategies for systematic reviews published in high-quality peer-reviewed journals and registered systematic review protocols [27-35] as well as registered systematic review protocols $[36,37]$ where SES was the primary exposure of interest. The ACSC block of terms included both general ACSC terms (e.g. avoidable hospitalization) and terms for individual chronic ACSCs (iron-deficiency anemia, angina, atrial fibrillation and flutter, asthma, COPD, CHF, epilepsy and seizures, diabetes and diabetic complications, and 
hypertension). This ensured that studies of individual chronic ACSCs that did not specifically describe them as conditions sensitive to ambulatory care were still captured in the search. General terms were identified from keywords listed in abstracts of peer-reviewed articles related to ACSCs as well as published systematic reviews [21]. Previous Cochrane reviews of condition-specific outcomes were consulted to generate appropriate search strategies for each condition [38-51]. Exclusion filters for randomized controlled trials [52], countries classified as low-income to upper-middle-income economies according to the World Bank [53], articles published in languages other than English, articles published prior to 1990, and, where possible, publication sources other than peer-reviewed journals and books were then applied. Search strategies included known common alternative spellings (e.g. hospitalization vs hospitalisation), and were optimized for each database. See Additional file 1 for a search strategy exemplar.

\section{Study inclusion and exclusion criteria}

Eligibility criteria for included studies are listed in Table 1.

\section{Study selection procedure}

Studies were selected for inclusion using a two-stage process. First, titles and abstracts were independently screened by LEW and EDP. Full texts of screened articles were then independently evaluated for inclusion by LEW and EDP. Any conflicts were resolved by discussion. If consensus could not be achieved, then a final decision was made in discussion with LCR. Reference lists of final articles included after quality assessment were hand searched by LEW. Study selection was managed using Covidence, an online tool for systematic review management.

\section{Data extraction strategy}

A data extraction form was developed by LEW, piloted by LEW and EDP, and modified based on user findings and feedback. Data related to study characteristics, SES exposure, ACSC hospitalization outcome, and study results were extracted by LEW. Data extraction was managed using Microsoft Excel. See Additional file 2 for the data extraction form.

\section{Study quality assessment}

Study quality assessment was conducted by LEW using appropriate The Joanna Briggs Institute Critical Appraisal

Table 1 Study inclusion and exclusion criteria

\begin{tabular}{|c|c|}
\hline Component & Explanation \\
\hline Population & $\begin{array}{l}\text { Inclusion: Study population has a mean age } \pm 1 \text { SD }<75 \text { years of age. If this criteria could not be evaluated due to } \\
\text { missing data, the study was still included. } \\
\text { Exclusion: } \\
\text { - Pediatric studies were excluded during full-text review as both the concept and effect of SES on health outcomes } \\
\text { differs in childhood relative to adulthood, and thus these studies were determined to be out of scope. }\end{array}$ \\
\hline Exposure & $\begin{array}{l}\text { Inclusion: Individual- or area-level SES defined as income, education, occupation, and social class in accordance with } \\
\text { the WHO Commission on Social Determinants of Health conceptual framework. } \\
\text { Exclusion: } \\
\text { - SES was not the clear primary exposure of interest, including studies that did not include SES in their title or } \\
\text { objectives, evaluated multiple predictors of interest (e.g. person and health system characteristics), or adjusted } \\
\text { for SES as a confounding variable as these studies were not optimally designed to evaluate the effects of SES, } \\
\text { decreasing interpretability of effect sizes. } \\
\text { Exception: Studies that only included demographic and health status covariates as these are potential SES } \\
\text { confounding variables, and SES effect sizes could be reasonably interpreted. } \\
\text { - Only proxy measures for SES were used (e.g. car ownership, insurance status). }\end{array}$ \\
\hline Outcome & $\begin{array}{l}\text { Inclusion: Hospitalization, including emergency department visits, for chronic ACSCs. Both aggregate and condition- } \\
\text { specific outcomes were included. Studies were included if aggregate outcomes included other ACSCs in addition to } \\
\text { chronic ACSCs listed in this review. } \\
\text { Exclusion: } \\
\text { - Sole study outcome was length of stay or hospital readmissions as readmitted individuals were considered at } \\
\text { greater risk of hospitalization relative to the general population. } \\
\text { - Selected chronic ACSCs were narrowed during full-text review, excluding iron-deficiency anemia and atrial } \\
\text { fibrillation and flutter, to be consistent with the Canadian definition (ie. angina, asthma, CHF, COPD, diabetes } \\
\text { and diabetic complications, epilepsy and seizures, and hypertension). } \\
\text { - Outcome was risk of hospitalization for an ACSC relative to a non-ACSC. }\end{array}$ \\
\hline Study Setting & Inclusion: Countries with high-income economies. \\
\hline Study Design & $\begin{array}{l}\text { Inclusion: Observational (i.e. cross-sectional, case-control, or cohort). } \\
\text { Exclusion: } \\
\text { - Purely descriptive studies that did not clearly articulate the SES-ACSC relationship using measures of effect } \\
\text { (i.e. risk ratio, rate ratio, odds ratio, hazard ratio, Relative Index of Inequality, Slope Index of Inequality). }\end{array}$ \\
\hline Publication & $\begin{array}{l}\text { Inclusion: } \\
\text { - Written in the English language. } \\
\text { - Published between January 1, } 1990 \text { to July 31, } 2018 . \\
\text { - Published in a peer-reviewed journal. }\end{array}$ \\
\hline
\end{tabular}


Tools for a given observational study design [54]. Studies were appraised for selection and description of study participants, valid and reliable measurements of exposure and outcome, identification and control of potential confounders, and use of statistical methods. Studies that calculated rate ratios using standardized rates without additional adjustment for other confounders were separately synthesized as studies with minimal adjustment (ie. use of age- or age- and sex-standardized rates to calculate measures of association).

\section{Data synthesis and presentation}

Included studies were qualitatively synthesized by type of SES exposure. Within each exposure type, results from studies with full adjustment for potential confounding variables were separately described from those with minimal adjustment (ie. use of age- or age- and sex-standardized rates to calculate measures of association). Given variation in measures of effect used, limiting cross-interpretation of effect sizes, the overall pattern of association between exposure and outcome were narratively described for each study. No additional analyses were conducted.

\section{Protocol and registration PROSPERO CRD42018088727.}

\section{Results}

\section{Review statistics}

Of the 15,857 unique peer-reviewed studies identified, 31 studies met the eligibility criteria and were of sufficient quality for inclusion (Fig. 1) (Table 2). Study location was distributed across 11 different countries in North America $(n=14)$, Europe $(n=14)$, Oceania $(n=2)$, and Asia $(n=1)$. Both aggregate and condition-specific
ACSC outcomes were reported (Fig. 2a), and multiple SES constructs were used including income, deprivation, education, and occupation (Fig. 2b). The majority of studies used group-level rather than individual-level SES exposure variables. All studies were either cohort or cross-sectional studies with almost half of the studies $(n=13)$ conducting a minimally adjusted analysis using standardized rates.

\section{Effect of SES on ACSC hospitalization outcomes}

The majority of studies identified that lower SES was significantly associated with a higher risk of ACSC hospitalization, regardless of the SES exposure or ACSC hospitalization outcome measured (Table 3).

\section{Income}

Of the 12 studies with full adjustment for confounding variables, 11 studies found that lower income was associated with a higher risk or rate of ACSC hospitalizations in their fully adjusted models with 10 of these studies reporting significant effects. In contrast, one study of the effect of income on epilepsy hospitalizations and ER visits found that lower income decreased odds of hospitalizations and ER visits after adjusting for treatment site, but the effect was not significant [59]. Four minimally adjusted analyses also found that lower income significantly increased risk of hospitalization with only one study reporting a non-significant relationship between income and rate of COPD hospitalizations [71].

\section{Education}

All six fully adjusted studies found that lower education is associated with higher risk of ACSC hospitalization with three of these studies reporting significant associations. One study of the effect of education on asthma hospitalizations or ED visits reported conflicting results when using Poisson versus logistic regression [57]. Two

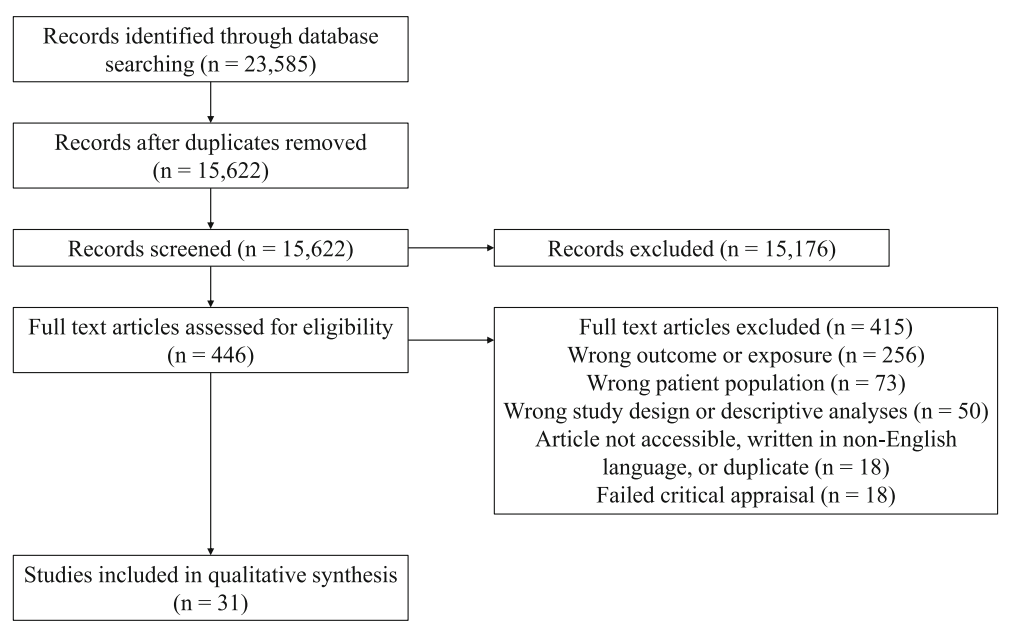

Fig. 1 Study selection flow diagram 


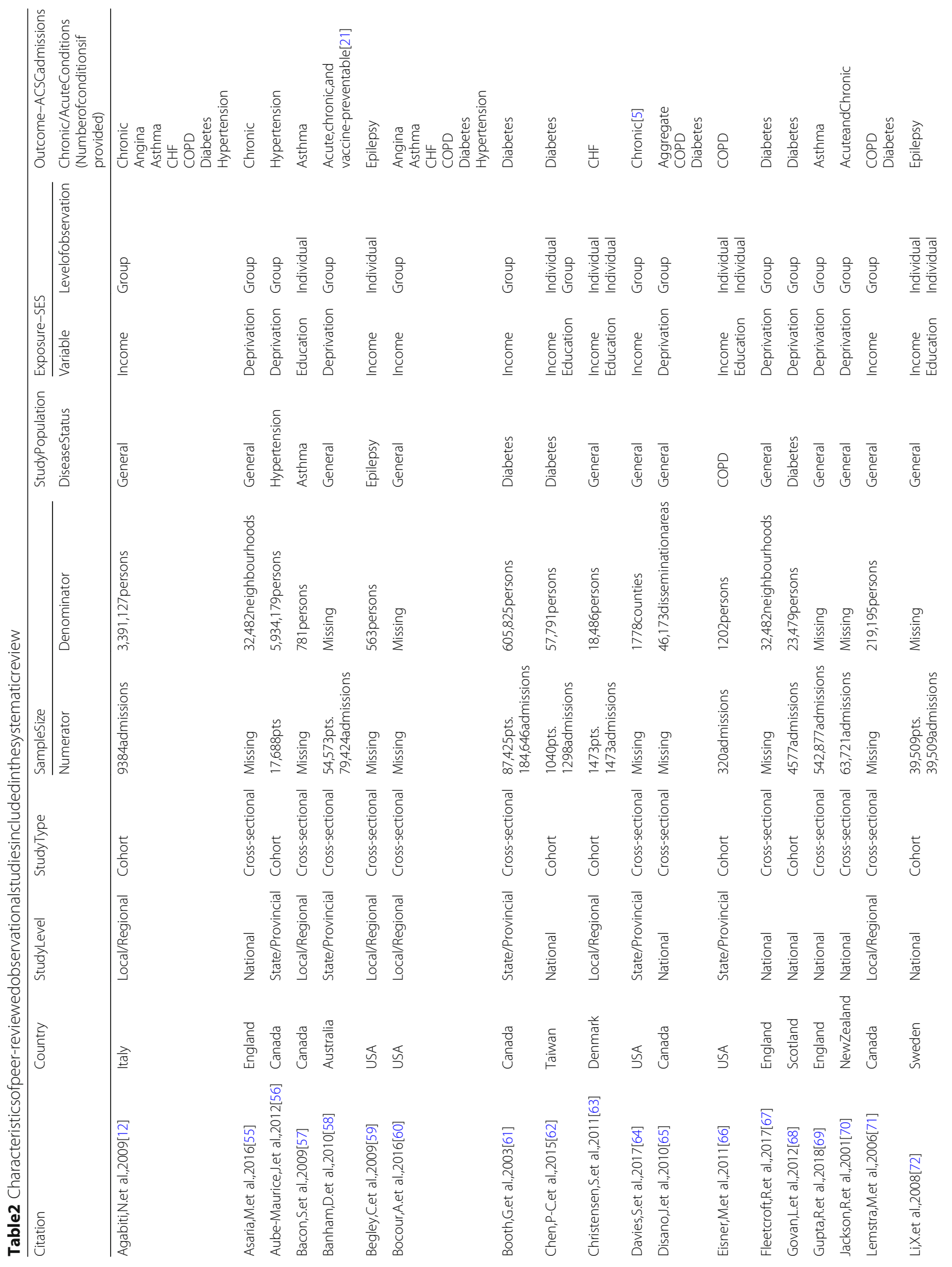




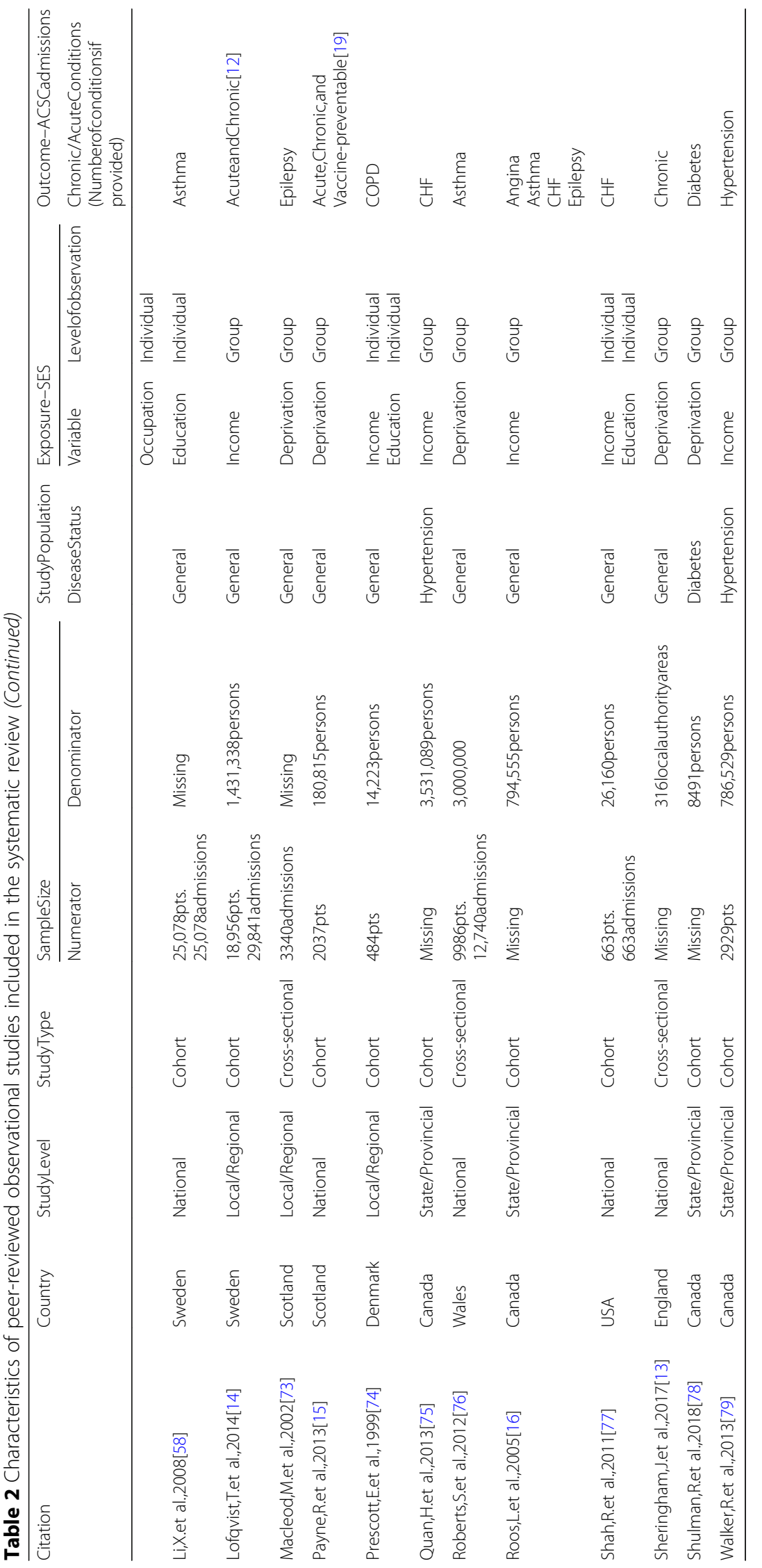




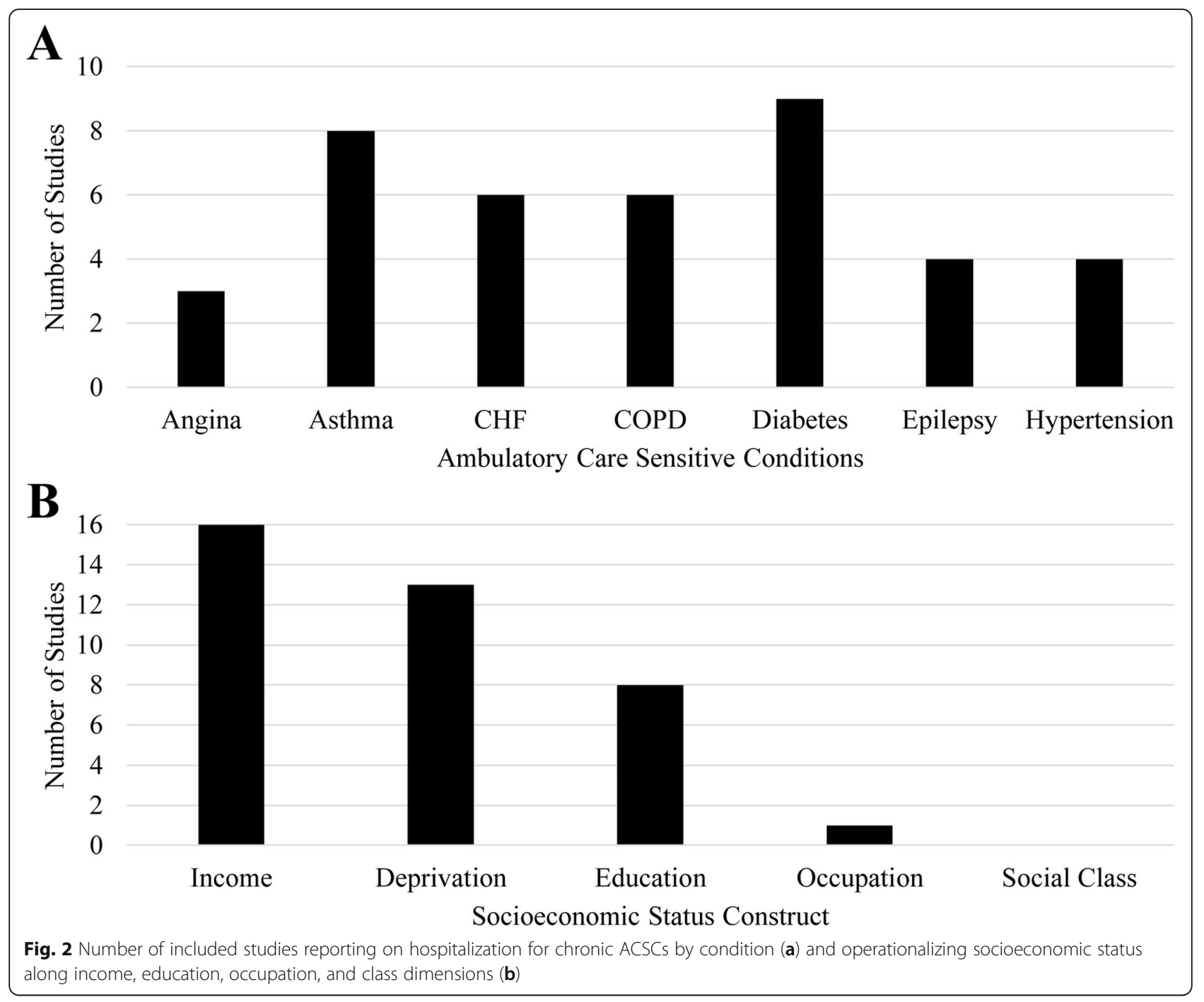

minimally adjusted analyses similarly found significant associations between lower education and increased incidence of asthma and epilepsy hospitalizations.

\section{Occupation}

The sole minimally adjusted study of the effect of occupational class on incidence of epilepsy hospitalizations observed that lower occupational class is weakly associated with higher ACSC hospitalization rates [72].

\section{Deprivation}

All five fully adjusted studies observed that higher deprivation is associated with higher risk or rate of ACSC hospitalizations. Eight minimally adjusted analyses reported the same findings, four of which were significant and four could not be determined due to missing confidence interval information.

\section{Quality assessment}

Forty-nine studies were critically appraised on characteristics that could introduce different biases including study population selection, exposure and outcome measurement, loss to follow up (cohort studies), identification and control of potential confounding variables, and usage of appropriate statistical methods. Of the 49 studies, 18 were excluded due to poor quality assessments. A number of studies $(n=11)$ used linear regression to model rate outcomes. This can be seen as inappropriate since linear regression assumes an incorrect error distribution for rate outcomes, which violates model assumptions. Two studies used other inappropriate methodologies, including choice of an outdated standard population for standardizing hospitalization rates and treating longitudinal data of the same study population as independent observations. Three studies lacked sufficient methodological information required for critical appraisal. One study used an appropriate regression model but selected variables using 
Table 3 Associations between socioeconomic status constructs and ambulatory care sensitive hospitalization outcomes in included studies $(n=31)$

\begin{tabular}{|c|c|c|c|c|c|}
\hline Citation & $\begin{array}{l}\text { Measure of } \\
\text { Association }\end{array}$ & ACSC & $\begin{array}{l}\text { Effect Size; by model } \\
\text { adjustment if provided }\end{array}$ & $\begin{array}{l}95 \% \\
\text { Confidence } \\
\text { Interval }\end{array}$ & $\begin{array}{l}\text { Direction of Association } \\
\& \text { Interpretation }(+/-)\end{array}$ \\
\hline
\end{tabular}

\section{Income - Fully Adjusted Analyses}

Agabiti, N.

et al., 2009 [12]

Rate Ratio

Lowest income

quintile / Highest

income quintile)

Begley, C.

et al., 2009 [59]

Odds Ratio (Income

$<100 \%$ of federal

poverty level quartile

/ Income $\geq 400 \%$ of

federal poverty level

quartile

Booth, G.

et al., 2003 [61]

Odds Ratio

Unadjusted: (Lowest

income quintile /

Highest income

quintile)

Adjusted: Per decline

in income quintile

Chen, P-C

Odds Ratio

et al., 2015 [62] (Low income quartile

/ Highest income

quartile)

Christensen, S.

et al., 2011 [63]

Hazard Ratio

(High income tertile /

Low income tertile)

Davies, S.

et al., 2017 [64]

Rate Ratio

(Highest decile of percent population below FPL / Lowest decile of percent population below FPL)

(10th percentile of median income / 90th percentile of median income)

Eisner, $\mathrm{M}$. Hazard Ratio

et al., 2011 [56]

income tertile

High income tertile)

Lofqvist, T.

et al., 2014 [14]

Odds Ratio

(Lowest income

quintile / Highest

income quintile)

$\begin{array}{lll}\text { Chronic }(n=6) & 2.59 & 2.35-2.85 \\ \text { Angina } & 1.97 & 1.70-2.30 \\ \text { Asthma } & 2.37 & 1.84-3.04 \\ \text { CHF } & 3.78 & 3.09-4.62 \\ \text { COPD } & 4.23 & 3.37-5.31 \\ \text { Diabetes } & 2.77 & 2.29-3.36 \\ \text { Hypertension } & 1.64 & 1.31-2.04\end{array}$

Epilepsy

Hospitalizations:

4.7 (Unadjusted)

2.9 (Adjusted for age, sex

and clinical characteristics)

0.8 (Additionally adjusted

for treatment site)

ER visits:

3.0 (Unadjusted)

2.2 (Adjusted for age, sex

and clinical characteristics)

0.5 (Additionally adjusted

for treatment site)

Diabetes

1.43 (Unadjusted)

1.09 (Adjusted for age, sex,

rurality, comorbidity,

frequency of physician

visits, continuity of care

physician speciality, and

geographic region)

Diabetes $\quad 2.89$ (Adjusted for age, sex time of diabetes diagnosis, comorbidities, participation

in P4P program, education, and urbanization)

2.44 (Additionally adjusted

for health care provider

ownership and level)

$\mathrm{CHF}$

0.67 (Female - Adjusted for age and time period)

0.66 (Male - Adjusted for age and time period)

$1.4-15.9$
$0.9-9.9$

$0.2-3.3$

(-) As income decreases, hospitalization rate increases. odds of ER visits and odds of hospitalization increases. Adjustment for treatment site mitigates income effect.

$1.6-5.7$

$1.1-4.3$

$0.2-1.4$

$1.40-1.46$

$1.08-1.10$

(-) As income decreases, odds of hospitalization or ED visits increases.

(-) As income decreases, odds of hospitalization increases.

$0.51-0.89$

$0.42-0.66$

Chronic

1.91 (Percent below poverty line)

1.44 (Median household income)

1.50 (Percent below poverty line)

$1.78-2.04$

Chronic

Asthma

1.19 (Median household income)

$1.35-1.53$

$1.39-1.62$

$1.11-1.27$
(-) As income increases, hospitalization risk decreases.

(+) As percent below poverty line increases, ED visit risk increases. (-) As income decreases, ED visit risk increases.

\section{COPD}

Acute and chronic
2.9 (Adjusted for age, sex, race, and education)

2.1 (Additionally adjusted for smoking history, occupational exposures, BMI, and co-morbidities) 1.5 (Additionally adjusted for COPD severity)

Ages 18-64:

1.52 (Adjusted for age and sex) $\quad 1.44-1.60$

1.12 (Additionally adjusted for marital 1.06-1.19 status, country of birth, education,
(-) As income decreases, hospitalization rate increases.
(-) As income decreases, hospitalization or ED visit risk increases. 
Table 3 Associations between socioeconomic status constructs and ambulatory care sensitive hospitalization outcomes in included studies $(n=31)$ (Continued)

\begin{tabular}{|c|c|c|c|c|c|}
\hline Citation & $\begin{array}{l}\text { Measure of } \\
\text { Association }\end{array}$ & ACSC & $\begin{array}{l}\text { Effect Size; by model } \\
\text { adjustment if provided }\end{array}$ & $\begin{array}{l}95 \% \\
\text { Confidence } \\
\text { Interval }\end{array}$ & $\begin{array}{l}\text { Direction of Association } \\
\& \text { Interpretation }(+/-)\end{array}$ \\
\hline & & & $\begin{array}{l}\text { gainful employment, sickness benefit, } \\
\text { and social assistance) } \\
\text { Ages } 65-79: \\
1.28 \text { (Adjusted for age and sex) } \\
1.06 \text { (Additionally adjusted for marital } \\
\text { status, country of birth, education, } \\
\text { and social assistance) }\end{array}$ & $\begin{array}{l}1.21-1.36 \\
1.00-1.13\end{array}$ & \\
\hline $\begin{array}{l}\text { Prescott, E. } \\
\text { et al., } 1999 \text { [74] }\end{array}$ & $\begin{array}{l}\text { Hazard Ratio } \\
\text { (High income tertile / } \\
\text { Low income tertile) }\end{array}$ & COPD & $\begin{array}{l}\text { Male: } \\
0.30 \text { (Adjusted for age) } \\
0.32 \text { (Additionally adjusted for } \\
\text { smoking status, inhalation, and } \\
\text { duration of smoking) } \\
\text { Female: } \\
0.63 \text { (Adjusted for age) } \\
0.59 \text { (Additionally adjusted for } \\
\text { smoking status, inhalation, and } \\
\text { duration of smoking) }\end{array}$ & $\begin{array}{l}0.40-1.01 \\
0.37-0.95\end{array}$ & $\begin{array}{l}\text { (-) As income increases, } \\
\text { hospitalization risk } \\
\text { decreases. }\end{array}$ \\
\hline $\begin{array}{l}\text { Quan, H. } \\
\text { et al., } 2013 \text { [75] }\end{array}$ & $\begin{array}{l}\text { Hazard Ratio } \\
\text { (Highest income quintile / } \\
\text { Lowest income quintile) }\end{array}$ & $\mathrm{CHF}$ & 0.72 & $0.71-0.73$ & $\begin{array}{l}\text { (-) As income increases, } \\
\text { hospitalization risk } \\
\text { decreases. }\end{array}$ \\
\hline $\begin{array}{l}\text { Shah, R. } \\
\text { et al., } 2011 \text { [77] }\end{array}$ & $\begin{array}{l}\text { Hazard Ratio } \\
\text { (Lowest income } \\
\text { quartile / Highest } \\
\text { income quartile) }\end{array}$ & $\mathrm{CHF}$ & $\begin{array}{l}3.43 \text { (Unadjusted) } \\
2.60 \text { (Adjusted for age, race/ } \\
\text { ethnicity, marital status, and } \\
\text { treatment assignments) } \\
1.56 \text { (Additionally adjusted for } \\
\text { clinical characteristics, health } \\
\text { behaviours, and insurance) }\end{array}$ & $\begin{array}{l}2.68-4.38 \\
2.01-3.37 \\
1.19-2.04\end{array}$ & $\begin{array}{l}\text { (-) As income decreases, } \\
\text { hospitalization risk } \\
\text { increases. }\end{array}$ \\
\hline $\begin{array}{l}\text { Walker, R. } \\
\text { et al., } 2013 \text { [79] }\end{array}$ & $\begin{array}{l}\text { Odds Ratio } \\
\text { (Highest income } \\
\text { quintile / Lowest } \\
\text { income quintile) }\end{array}$ & Hypertension & 0.59 & $0.51-0.68$ & $\begin{array}{l}\text { (-) As income increases, } \\
\text { odds of hospitalization } \\
\text { decreases. }\end{array}$ \\
\hline \multicolumn{6}{|c|}{ Income - Minimally Adjusted Analyses } \\
\hline $\begin{array}{l}\text { Bocour, A. } \\
\text { et al., } 2016 \text { [60] }\end{array}$ & $\begin{array}{l}\text { Rate Ratio } \\
\text { (Very high poverty } \\
\text { / Low poverty) }\end{array}$ & $\begin{array}{l}\text { Angina } \\
\text { Asthma } \\
\text { CHF } \\
\text { COPD } \\
\text { Diabetes } \\
\text { Hypertension }\end{array}$ & $\begin{array}{l}2.89 \\
5.35 \\
2.61 \\
3.30 \\
3.50 \\
3.03\end{array}$ & Missing & $\begin{array}{l}\text { (+) As poverty increases, } \\
\text { hospitalization rate } \\
\text { increases. }\end{array}$ \\
\hline $\begin{array}{l}\text { Lemstra, M. } \\
\text { et al., } 2006 \text { [71] }\end{array}$ & $\begin{array}{l}\text { Rate Ratio } \\
\text { (Low income / } \\
\text { Affluent) } \\
\text { (Dichotomous) }\end{array}$ & $\begin{array}{l}\text { COPD } \\
\text { Diabetes }\end{array}$ & $\begin{array}{l}1.53 \\
12.86\end{array}$ & $\begin{array}{l}0.88-2.67 \\
5.42-30.51\end{array}$ & $\begin{array}{l}\text { (-) As income decreases, } \\
\text { hospitalization rate } \\
\text { increases. }\end{array}$ \\
\hline $\begin{array}{l}\text { Li, X. et al., } \\
2008 \text { [72] }\end{array}$ & $\begin{array}{l}\text { Standardized } \\
\text { Incidence Ratio } \\
\text { (Low income tertile / } \\
\text { All economically } \\
\text { active persons }\end{array}$ & Epilepsy & $\begin{array}{l}1.13 \text { (Males) } \\
1.10 \text { (Females) }\end{array}$ & $\begin{array}{l}1.11-1.15 \\
1.07-1.12\end{array}$ & $\begin{array}{l}\text { (-) As income decreases, } \\
\text { hospitalization rate } \\
\text { increases. }\end{array}$ \\
\hline $\begin{array}{l}\text { Roos, L. et al., } \\
2005 \text { [16] }\end{array}$ & $\begin{array}{l}\text { Rate Ratio } \\
\text { (Lowest income } \\
\text { quintile / Highest } \\
\text { income quintile) }\end{array}$ & $\begin{array}{l}\text { Angina } \\
\text { Asthma } \\
\text { CHF } \\
\text { Epilepsy }\end{array}$ & $\begin{array}{l}1.39 \\
2.90 \\
1.73 \\
2.98\end{array}$ & $\begin{array}{l}1.21-1.58 \\
2.50-3.37 \\
1.58-1.92 \\
2.17-4.36\end{array}$ & $\begin{array}{l}(-) \text { As income decreases, } \\
\text { hospitalization rate } \\
\text { increases. }\end{array}$ \\
\hline \multicolumn{6}{|c|}{ Education - Fully Adjusted Analyses } \\
\hline $\begin{array}{l}\text { Bacon, S. et al., } \\
2009 \text { [57] }\end{array}$ & $\begin{array}{l}\text { Risk Ratio } \\
(<12 \text { years of } \\
\text { education } / \geq 12 \\
\text { years of education) } \\
\text { Odds Ratio } \\
\text { ( }<12 \text { years of }\end{array}$ & Asthma & $\begin{array}{l}0.93 \text { (Adjusted for age, sex, } \\
\text { and asthma severity) } \\
0.95 \text { (Additionally adjusted for } \\
\text { current smoking, BMl, and having } \\
\text { a mood and/or anxiety disorder) } \\
1.55 \text { (Adjusted for age, sex, and }\end{array}$ & $\begin{array}{l}0.90-0.97 \\
0.91-0.99 \\
1.02-2.27\end{array}$ & $\begin{array}{l}\text { (+) As education decreases, } \\
\text { risk of ED visits and } \\
\text { hospitalizations increases. } \\
(-) \text { As education decreases, } \\
\text { odds of ED visits and } \\
\text { hospitalizations increases. }\end{array}$ \\
\hline
\end{tabular}


Table 3 Associations between socioeconomic status constructs and ambulatory care sensitive hospitalization outcomes in included studies $(n=31)$ (Continued)

\begin{tabular}{|c|c|c|c|c|c|}
\hline Citation & $\begin{array}{l}\text { Measure of } \\
\text { Association }\end{array}$ & ACSC & $\begin{array}{l}\text { Effect Size; by model } \\
\text { adjustment if provided }\end{array}$ & $\begin{array}{l}95 \% \\
\text { Confidence } \\
\text { Interval }\end{array}$ & $\begin{array}{l}\text { Direction of Association } \\
\& \text { Interpretation }(+/-)\end{array}$ \\
\hline & & & \multicolumn{2}{|l|}{$\begin{array}{l}\text { smoking, BMI, and having a mood } \\
\text { and/or anxiety disorder) }\end{array}$} & \\
\hline $\begin{array}{l}\text { Chen, P-C. } \\
\text { et al., } 2015 \text { [62] }\end{array}$ & $\begin{array}{l}\text { Odds Ratio } \\
\text { (Lowest \% of } \\
\text { individuals with } \\
\text { higher education } \\
\text { quartile / Highest \% } \\
\text { of individuals with } \\
\text { higher education } \\
\text { quartile) }\end{array}$ & Diabetes & $\begin{array}{l}1.33 \text { (Adjusted for age, sex, time } \\
\text { of diabetes diagnosis, comorbidities, } \\
\text { participation in P4P program, income, } \\
\text { and urbanization) } \\
1.32 \text { (Additionally adjusted for health } \\
\text { care provider ownership and level) }\end{array}$ & $1.10-1.61$ & $\begin{array}{l}\text { (-) As education decreases, } \\
\text { odds of hospitalization } \\
\text { increases. }\end{array}$ \\
\hline $\begin{array}{l}\text { Christensen, S. } \\
\text { et al., } 2011 \text { [63] }\end{array}$ & $\begin{array}{l}\text { Hazard Ratio } \\
(>10 \text { years of } \\
\text { education tertile / } \\
<8 \text { years of } \\
\text { education tertile) }\end{array}$ & $\mathrm{CHF}$ & $\begin{array}{l}0.50 \text { (Female - Adjusted for age and } \\
\text { time period) } \\
0.53 \text { (Male - Adjusted for age and } \\
\text { time period) } \\
0.52 \text { (All - Adjusted for age, sex, } \\
\text { and time period) } \\
0.61 \text { (Additionally adjusted for } \\
\text { clinical characteristics, BMl, smoking, } \\
\text { and physical inactivity) }\end{array}$ & $\begin{array}{l}0.37-0.69 \\
0.42-0.66 \\
0.43-0.63 \\
0.50-0.73\end{array}$ & $\begin{array}{l}\text { (-) As education increases, } \\
\text { hospitalization risk } \\
\text { decreases. }\end{array}$ \\
\hline $\begin{array}{l}\text { Eisner, M. } \\
\text { et al., } 2011[66]\end{array}$ & $\begin{array}{l}\text { Hazard Ratio } \\
\text { (Less than high } \\
\text { school education } \\
\text { tertile / Post- } \\
\text { secondary education } \\
\text { completed tertile) }\end{array}$ & COPD & $\begin{array}{l}1.9 \text { (Adjusted for age, sex, race, } \\
\text { and education) } \\
1.5 \text { (Additionally adjusted for smoking } \\
\text { history, occupational exposures, BMI, } \\
\text { and co-morbidities) } \\
1.1 \text { (Additionally adjusted for } \\
\text { COPD severity) }\end{array}$ & $\begin{array}{l}1.3-2.7 \\
1.01-2.1 \\
0.7-1.6\end{array}$ & $\begin{array}{l}\text { (-) As education decreases, } \\
\text { risk of hospitalization or ED } \\
\text { visit increases. }\end{array}$ \\
\hline $\begin{array}{l}\text { Prescott, E. } \\
\text { et al., } 1999 \text { [74] }\end{array}$ & $\begin{array}{l}\text { Hazard Ratio } \\
(>11 \text { years of } \\
\text { education tertile / <8 } \\
\text { years of education } \\
\text { tertile) }\end{array}$ & COPD & $\begin{array}{l}\text { Male: } 0.44 \text { (Adjusted for age) } \\
0.55 \text { (Additionally adjusted for } \\
\text { smoking status, inhalation, and } \\
\text { duration of smoking) } \\
\text { Female: } \\
0.27 \text { (Adjusted for age) } \\
0.28 \text { (Additionally adjusted for } \\
\text { smoking status, inhalation, and } \\
\text { duration of smoking) }\end{array}$ & $\begin{array}{l}0.27-0.72 \\
0.34-0.90\end{array}$ & $\begin{array}{l}\text { (-) As education increases, } \\
\text { hospitalization risk } \\
\text { decreases. }\end{array}$ \\
\hline $\begin{array}{l}\text { Shah, R. } \\
\text { et al., } 2011 \text { [77] }\end{array}$ & $\begin{array}{l}\text { Hazard Ratio } \\
\text { (Less than high } \\
\text { school tertile / Post- } \\
\text { secondary completed } \\
\text { tertile) }\end{array}$ & $\mathrm{CHF}$ & $\begin{array}{l}2.01 \text { (Unadjusted) } \\
1.96 \text { (Adjusted for age, race/ } \\
\text { ethnicity, marital status, and } \\
\text { treatment assignments) } \\
1.21 \text { (Additionally adjusted for } \\
\text { clinical characteristics, health } \\
\text { behaviours, and insurance) }\end{array}$ & $\begin{array}{l}1.53-2.65 \\
1.48-2.60 \\
0.90-1.62\end{array}$ & $\begin{array}{l}\text { (-) As education decreases, } \\
\text { hospitalization risk } \\
\text { increases. }\end{array}$ \\
\hline \multicolumn{6}{|c|}{ Education - Minimally Adjusted Analyses } \\
\hline $\begin{array}{l}\text { Li, X. et al., } \\
2008 \text { [72] }\end{array}$ & $\begin{array}{l}\text { Standardized Incidence Ratio } \\
\text { ( } \leq 9 \text { years of } \\
\text { education tertile / All } \\
\text { economically active } \\
\text { persons) }\end{array}$ & Asthma & $\begin{array}{l}1.03 \text { (Male) } \\
1.05 \text { (Female) }\end{array}$ & $\begin{array}{l}1.01-1.05 \\
1.03-1.08\end{array}$ & $\begin{array}{l}\text { (-) As education decreases, } \\
\text { hospitalization rate } \\
\text { increases. }\end{array}$ \\
\hline $\begin{array}{l}\text { Li, X. et al., } \\
2008 \text { [72] }\end{array}$ & $\begin{array}{l}\text { Standardized Incidence Ratio } \\
\text { ( } \leq 9 \text { years of } \\
\text { education tertile / All } \\
\text { economically active } \\
\text { persons) }\end{array}$ & Epilepsy & $\begin{array}{l}1.06 \text { (Male) } \\
1.06 \text { (Female) }\end{array}$ & $\begin{array}{l}1.04-1.08 \\
1.04-1.08\end{array}$ & $\begin{array}{l}\text { (-) As education decreases, } \\
\text { hospitalization rate } \\
\text { increases. }\end{array}$ \\
\hline \multicolumn{6}{|c|}{ Occupation - Minimally Adjusted Analyses } \\
\hline $\begin{array}{l}\text { Li, X. et al., } \\
2008[72]\end{array}$ & $\begin{array}{l}\text { Standardized } \\
\text { Incidence Ratio } \\
\text { (Unskilled workers / }\end{array}$ & Epilepsy & $\begin{array}{l}1.04 \text { (Male) } \\
1.01 \text { (Female) }\end{array}$ & $\begin{array}{l}1.02-1.06 \\
0.99-1.03\end{array}$ & $\begin{array}{l}\text { (-) As occupation } \\
\text { decreases, hospitalization } \\
\text { rate increases. }\end{array}$ \\
\hline
\end{tabular}


Table 3 Associations between socioeconomic status constructs and ambulatory care sensitive hospitalization outcomes in included studies $(n=31)$ (Continued)

\begin{tabular}{|c|c|c|c|c|c|}
\hline Citation & $\begin{array}{l}\text { Measure of } \\
\text { Association }\end{array}$ & ACSC & $\begin{array}{l}\text { Effect Size; by model } \\
\text { adjustment if provided }\end{array}$ & $\begin{array}{l}95 \% \\
\text { Confidence } \\
\text { Interval }\end{array}$ & $\begin{array}{l}\text { Direction of Association } \\
\& \text { Interpretation }(+/-)\end{array}$ \\
\hline \multicolumn{6}{|c|}{ Deprivation - Fully Adjusted Analyses } \\
\hline $\begin{array}{l}\text { Aube-Maurice, } \\
\text { J. et al., } 2012 \text { [56] }\end{array}$ & $\begin{array}{l}\text { Risk Ratio } \\
\text { (Most deprived } \\
\text { quintile / Least } \\
\text { deprived quintile) }\end{array}$ & Hypertension & $\begin{array}{l}\text { Males: } \\
1.29 \text { (Material deprivation) } \\
1.14 \text { (Social deprivation) } \\
\text { Females: } \\
1.60 \text { (Material deprivation) } \\
1.04 \text { (Social deprivation) }\end{array}$ & $\begin{array}{l}1.18-1.40 \\
1.05-1.24 \\
1.43-1.79 \\
0.93-1.16\end{array}$ & $\begin{array}{l}\text { (+) As deprivation increases, } \\
\text { hospitalization risk } \\
\text { increases. }\end{array}$ \\
\hline $\begin{array}{l}\text { Govan, L. } \\
\text { et al., } 2012 \text { [68] }\end{array}$ & $\begin{array}{l}\text { Odds Ratio } \\
\text { (Most deprived } \\
\text { quintile / Least } \\
\text { deprived quintile) }\end{array}$ & Diabetes & 2.82 & $2.33-3.42$ & $\begin{array}{l}\text { (+) As deprivation increases, } \\
\text { odds of hospitalization } \\
\text { increases. }\end{array}$ \\
\hline $\begin{array}{l}\text { Gupta, R. } \\
\text { et al., } 2018 \text { [69] }\end{array}$ & $\begin{array}{l}\text { Rate Ratio } \\
\text { (Most deprived } \\
\text { quintile / Least } \\
\text { deprived quintile) }\end{array}$ & Asthma & $\begin{array}{l}3.34 \text { (Ages 5-44) } \\
2.01 \text { (Ages 45-74) }\end{array}$ & $\begin{array}{l}3.30-3.38 \\
1.98-2.05\end{array}$ & $\begin{array}{l}\text { (+) As deprivation increases, } \\
\text { hospitalization rate } \\
\text { increases. }\end{array}$ \\
\hline $\begin{array}{l}\text { Payne, R. et al., } \\
2013 \text { [15] }\end{array}$ & $\begin{array}{l}\text { Odds Ratio } \\
\text { (Most deprived } \\
\text { quintile / Least } \\
\text { deprived quintile) }\end{array}$ & $\begin{array}{l}\text { Acute and } \\
\text { chronic }\end{array}$ & $\begin{array}{l}2.84 \text { (Unadjusted) } \\
1.98 \text { (Adjusted for age, sex, } \\
\text { multimorbidity, and mental } \\
\text { health condition) }\end{array}$ & $\begin{array}{l}2.40-3.37 \\
1.63-2.41\end{array}$ & $\begin{array}{l}\text { (+) As deprivation increases, } \\
\text { odds of hospitalization } \\
\text { increases. }\end{array}$ \\
\hline $\begin{array}{l}\text { Shulman, R. } \\
\text { et al., } 2018 \text { [78] }\end{array}$ & $\begin{array}{l}\text { Rate Ratio } \\
\text { (Most deprived } \\
\text { quintile / Least } \\
\text { deprived quintile) }\end{array}$ & Diabetes & $\begin{array}{l}\text { (Hospitalizations) } \\
\text { (ER visits) }\end{array}$ & $\begin{array}{l}\text { Values not } \\
\text { reported. See } \\
\text { Fig. } 2 \text { in } \\
\text { article. }\end{array}$ & $\begin{array}{l}\text { (+) As deprivation increases, } \\
\text { hospitalization and ER visit } \\
\text { rate increases. }\end{array}$ \\
\hline \multicolumn{6}{|c|}{ Deprivation - Minimally Adjusted Analyses } \\
\hline $\begin{array}{l}\text { Asaria, M. } \\
\text { et al., } 2016 \text { [55] }\end{array}$ & $\begin{array}{l}\text { RII } \\
\text { SII }\end{array}$ & Chronic & $\begin{array}{l}1.06 \\
6.07\end{array}$ & $\begin{array}{l}1.04-1.07 \\
5.97-6.16\end{array}$ & $\begin{array}{l}\text { (+) As deprivation increases, } \\
\text { hospitalization risk } \\
\text { increases. }\end{array}$ \\
\hline $\begin{array}{l}\text { Banham, D. } \\
\text { et al., } 2010 \text { [58] }\end{array}$ & $\begin{array}{l}\text { Rate ratio } \\
\text { (Most disadvantaged } \\
\text { quintile / Least } \\
\text { disadvantaged } \\
\text { quintile) }\end{array}$ & $\begin{array}{l}\text { Acute, chronic, } \\
\text { and vaccine- } \\
\text { preventable }\end{array}$ & 2.5 & $2.5-2.5$ & $\begin{array}{l}\text { (+) As deprivation increases, } \\
\text { hospitalization rate } \\
\text { increases. }\end{array}$ \\
\hline $\begin{array}{l}\text { Disano, J. } \\
\text { et al., } 2010 \text { [65] }\end{array}$ & $\begin{array}{l}\text { Rate Ratio } \\
\text { (Low SES tertile / } \\
\text { High SES tertile) }\end{array}$ & $\begin{array}{l}\text { Aggregate } \\
\text { COPD } \\
\text { Diabetes }\end{array}$ & $\begin{array}{l}2.6 \\
2.7 \\
3\end{array}$ & $\begin{array}{l}\text { Missing } \\
\text { Missing } \\
\text { Missing }\end{array}$ & $\begin{array}{l}\text { (+) As deprivation increases, } \\
\text { hospitalization rate } \\
\text { increases. }\end{array}$ \\
\hline $\begin{array}{l}\text { Fleetcroft, R. } \\
\text { et al., } 2017 \text { [67] }\end{array}$ & $\begin{array}{l}\text { RII } \\
\text { SII }\end{array}$ & Diabetes & $\begin{array}{l}1.18 \\
84.25\end{array}$ & $\begin{array}{l}1.15-1.22 \\
81.62-86.88\end{array}$ & $\begin{array}{l}\text { (+) As deprivation increases, } \\
\text { hospitalization risk } \\
\text { increases. }\end{array}$ \\
\hline $\begin{array}{l}\text { Jackson, R. } \\
\text { et al., } 2001 \text { [70] }\end{array}$ & $\begin{array}{l}\text { Rate Ratio } \\
\text { (Most deprived 10\% / } \\
\text { Least deprived 40\%) }\end{array}$ & Aggregate & 2.3 & Missing & $\begin{array}{l}\text { (+) As deprivation increases, } \\
\text { hospitalization rate } \\
\text { increases. }\end{array}$ \\
\hline $\begin{array}{l}\text { Macleod, M. } \\
\text { et al., 2002 [73] }\end{array}$ & $\begin{array}{l}\text { Rate Ratio } \\
\text { (Most deprived septile } \\
\text { / Least deprived } \\
\text { septile) }\end{array}$ & Epilepsy & 3.30 & Missing & $\begin{array}{l}\text { (+) As deprivation increases, } \\
\text { hospitalization rate } \\
\text { increases. }\end{array}$ \\
\hline $\begin{array}{l}\text { Roberts, S. } \\
\text { et al., } 2012 \text { [76] }\end{array}$ & $\begin{array}{l}\text { Rate Ratio } \\
\text { (Most deprived } \\
\text { quintile / Least } \\
\text { deprived quintile) }\end{array}$ & Asthma & 2.48 & $2.34-2.62$ & $\begin{array}{l}\text { (+) As deprivation increases, } \\
\text { hospitalization rate for } \\
\text { severe asthma increases. }\end{array}$ \\
\hline $\begin{array}{l}\text { Sheringham, J. } \\
\text { et al., } 2017 \text { [13] }\end{array}$ & SII & Aggregate & 5.98 & Missing & $\begin{array}{l}(+) \text { As deprivation increases, } \\
\text { hospitalization risk } \\
\text { increases. }\end{array}$ \\
\hline
\end{tabular}


backward stepwise selection, which can artificially select for variables with small $p$-values and yield a final model that does not appropriately adjust for SES confounding variables. Lastly, one cohort study had a high risk of loss-to-follow up, which can result in high risk of bias. Study specific quality assessments can be found in Additional files 3 and 4.

Of the 31 included studies, 15 studies conducted analyses that minimally adjusted for potential SES confounding variables (ie. use of age- or age- and sexstandardized rates to calculate measures of association) and thus had higher risk of residual confounding of their reported estimates. The remaining 18 studies were of sufficient quality with low risk of bias. However, potential biases in these studies were possible. Studies inferring individual-level SES using group-level exposure information $(n=11)$ are at risk of exposure misclassification. While most of these studies identified hospitalizations using registries, three studies used self-reported information which may be less accurate and subject to recall bias due to varying recall ability of study participants for when and why they were hospitalized. Lastly, studies varied in identification and control of potential confounding variables. While some studies presented sequentially adjusted models, others only presented fully adjusted models. Despite this variation, patterns of association within and across studies generally remained the same, although the magnitude of effect was attenuated after adjustment.

\section{Evidence of effectiveness}

In this review, almost all studies reported a relationship between low SES and high ACSC hospitalization outcomes with lower SES associated with higher risk of ACSC hospitalization outcomes across different SES exposures, aggregate and condition-specific outcomes, and study level. Two studies reporting a positive effect in their fully adjusted models were both local/regional studies of condition specific ACSC outcomes with limited sample sizes $(<800$ persons), and thus are of lower impact. Strong consistency of results provides evidence of an association between SES and ACSC hospitalizations with lower SES individuals at greater risk of hospitalization compared to higher SES individuals.

\section{Discussion}

\section{Summary of key findings}

This systematic review is the first to comprehensively identify and analyze peer-reviewed literature on the relationship between SES and chronic ACSC hospitalizations, considering both aggregate and condition-specific outcomes that are common to a number of international health systems that utilize ACSC hospitalization rates as an indicator of health system performance. The review determined that there is an increased risk of chronic ACSC hospitalizations associated with lower levels of SES and this finding is robustly observed across different SES exposure definitions, aggregate and condition-specific hospitalization outcomes, local to national observational levels, and varied geographic locations. Studies vary in their methodological approaches including identification and control of confounding variables and definition of outcomes. Inappropriate use of regression techniques to model hospitalization rate outcomes is not uncommon and limits interpretation of some study findings.

\section{Strengths and limitations}

This review specifically focused on chronic ACSC hospitalizations that are commonly measured in other international health systems that monitor ACSC hospitalization rates, increasing relevance of study findings. Distinguishing chronic from acute and vaccinepreventable ACSC hospitalizations is important as the impact of SES on hospitalization rates for these conditions likely differs between these groupings of conditions and should be separately summarized [80]. Chronic ACSC hospitalizations are of importance to both population health and primary health care as prevalence of chronic ACSCs, and correspondingly, size of populations at greater risk of hospitalization, is increasing within countries. A greater understanding of upstream risk factors, such as SES, is needed to help address this increasingly important population health, health system, and health equity outcome.

Our methodological approach had a number of strengths. First, the search strategy incorporated both general and condition-specific ACSC terms to increase sensitivity of the search as studies of condition-specific outcomes will not necessarily use generic ACSC terms (e.g. avoidable, preventable, ambulatory care sensitive) in their titles and abstracts. Consultation and usage of high-quality, published search strings for each condition also helped to successfully retrieve articles relating to these conditions. Use of both health and social science databases further ensured that studies of SES and ACSC hospitalization outcomes were identified. Lastly, this review specifically focused on studies where SES was the primary exposure of interest. SES is a very common independent variable that is included in studies of ACSC hospitalization outcomes. However, we distinguished between usage of SES as an exposure variable versus an adjustment or confounding variable. While this possibly decreased some eligible studies, this allowed us to more specifically interpret reported associations between SES and chronic ACSC hospitalizations by reviewing studies designed to explicitly assess this relationship. In 
addition, studies that were ambiguous on the role of SES as an exposure also tended to be of lower quality.

This review had several limitations that are important to acknowledge. First, this review was limited to peerreviewed literature, potentially missing grey literature evaluating the impact of SES on chronic ACSC hospitalizations. Second, we opted for more specific definitions of both SES and chronic ACSC hospitalizations in order to be more precise in the interpretation and aid in synthesis of results, potentially excluding studies using alternative SES definitions (e.g. insurance status, home ownership, car ownership). We specifically chose SES constructs that are commonly used across health systems in high-income economies to increase relevance. System-specific measures (e.g. insurance status in the US health system) and proxy measures of wealth were considered less relevant given the scope of this review. Likewise, a limited number of chronic ACSC hospitalizations were considered and did not include all possible chronic ACSCs evaluated by international health systems. We specifically chose conditions consistent with the Canadian definition that is used for health system performance monitoring. Further, these conditions are also measured by other similar health systems, and thus this limitation is not expected to greatly impact the relevance of this review in an international context. Importantly, we did not exclude studies using aggregate ACSC hospitalization outcomes that included both chronic and other ACSC conditions. While interpretation of studies using mixed aggregate outcomes was reduced, it more importantly prevented bias towards selection of studies evaluating condition-specific rather than aggregate outcomes. Lastly, we were unable to conduct a meta-analysis given the extensive heterogeneity in outcome definitions, exposure definitions, and measures of effect used.

\section{Heterogeneity in studies of SES and chronic ACSC hospitalizations}

There is inherent heterogeneity in how both SES and chronic ACSC hospitalizations are defined that precludes additional synthesis of results beyond narrative syntheses. In this review, SES was defined using multiple constructs. Even within a given construct, multiple definitions were used. For example, income constructs included area-level household income, individual-level household income, and percentage below federal poverty line. Deprivation was measured using multiple contextspecific indices including Index of Multiple Deprivation, Welsh Index of Multiple Deprivation, Scottish Index of Multiple Deprivation, and INSPQ Deprivation Index that measure both similar and unique aspects of deprivation, making cross-national generalizations difficult [81]. While education was most consistently defined, the number and meaning of categorical levels varied between studies. Likewise, definitions of chronic ACSC hospitalizations also varied including diagnostic codes used to identify conditions, inclusion of emergency department visits, hospital transfers, and hospital readmissions, and usage of age thresholds. Although we found consistent associations across different exposure and outcome definitions, this heterogeneity prevented additional meta-analyses of the effect of SES on chronic ACSC hospitalizations.

\section{Considerations for future studies}

There are a number of findings that have emerged from this review that are useful to consider for future research of SES and ACSC hospitalizations. Firstly, we note several challenges with the reporting of the effect of SES on ACSC hospitalization risk in the included observational studies. We recommend that researchers clarify the role that SES is playing in the analysis (i.e. as a primary exposure or not), which will impact how it is handled in the analysis. If SES is one of many covariates of interest, studies should publish models that sequentially and transparently adjust for different factors (e.g. demographic, socioeconomic, behavioural, health system) to facilitate interpretation of SES variables. If SES is a priori considered a confounding variable, then studies should consider how the SES effect size is interpreted as the model is not optimized for SES as an exposure. The ambiguity around the role of SES in the analysis of the included studies made summarizing the literature challenging.

Second, we recommend clarity in considering hospitalization outcomes. We found considerable variation in how hospitalization outcomes were defined. Specifically, there was confusion regarding whether allcause or cause-specific hospitalizations are measured, usage of diagnostic codes including whether primary only or primary and secondary codes were used, and whether readmissions were included. Further, we found variability in how studies described their comparator outcome group; specifically, indicating whether this group includes non-hospitalized individuals, individuals who are hospitalized but for a non-ACSC condition, or individuals who are hospitalized once for an ACSC condition. Lack of clarity in the comparison group prevents the ability to do meta-analysis in future reviews.

Third, we recommend more consistent reporting of study population characteristics. This was commonly missing, particularly in cross-sectional studies. These characteristics include: number of persons hospitalized, number of hospitalizations, age and sex distribution, and denominator information. Additional methodological considerations include using Poisson or negative binomial regression versus linear regression to model rate outcomes and avoiding use of stepwise selection of variables that may eliminate important confounders and 
affect standard errors. Stratification of results by age groups (e.g. pediatric, adult, and elderly) was also not commonly done but may be useful as the concept of both SES and avoidable hospitalizations are different within each of these groups.

With respect to future areas of research, we note that additional research was lacking in three important areas. First, while this review focused on chronic conditions, systematic reviews of SES as a risk factor for ACSC hospitalizations for acute and vaccine-preventable conditions were much more limited and would be needed for a more comprehensive understanding [80]. Second, more studies that specifically focus on elucidating the mechanisms between SES and ACSC hospitalizations. This includes the potential of SES as an indirect risk factor that is mediated through more proximal factors. Lastly, there are few studies that examine the delivery and impact of interventions for policies that may mitigate the impact of lower SES on risk of hospitalization by either directly improving SES conditions or through healthcare approaches.

\section{Conclusions}

This systematic review found a robust and consistent association between lower levels of SES and higher risk of chronic ACSC hospitalizations. Given the population health and primary health care importance of reducing these hospitalizations, an understanding and focus on upstream risk factors such as SES is warranted. The consistent and persistent SES inequalities observed in this review suggest that much work remains to reduce hospitalization risk among lower SES individuals. Additional evidence is needed on effective interventions for reducing overall hospitalization rates and reducing the inequality gap between different SES groups.

\section{Supplementary information}

Supplementary information accompanies this paper at https://doi.org/10. 1186/s12939-020-01160-0.

Additional file 1. EMBASE Search Strategy. Search strategy used to identify articles in the EMBASE database including search terms, search strings, and filters.

Additional file 2. Data Extraction Form. Form used to extract data from eligible full-text articles.

Additional file 3. Critical Appraisal of Included Articles ( $n=31$ ) using The Joanna Briggs Institute Critical Appraisal Tools. Study quality assessment results for each article included in the systematic review from eligible full-text articles.

Additional file 4. Critical Appraisal of Articles Excluded after Appraisal ( $n=18$ ) using The Joanna Briggs Institute Critical Appraisal Tools. Study quality assessment results for each article excluded upon appraisal from eligible full-text articles.

\section{Abbreviations}

ACSC: Ambulatory care sensitive condition; CHF: Congestive heart failure; COPD: Chronic obstructive pulmonary disease; INSPQ: Institut national de santé publique du Québec; SES: Socioeconomic status

\section{Acknowledgements}

The authors thank Catherine Bornbaum for her input on designing the search protocol, and Jessica Wong for her input on designing the search protocol and data extraction form.

\section{Authors' contributions}

LEW designed and conducted the search protocol, screened articles, extracted data, conducted critical appraisal, and wrote the manuscript. EDP screened articles. LCR designed the search protocol and wrote the manuscript. All authors read and approved the final manuscript.

\section{Funding}

This funding is supported by a Canadian Institutes for Health Research Operating Grant (FRN-142498). LCR is supported by a Canada Research Chair in Population Health Analytics. The funding bodies had no role in study design, data collection, analysis, and interpretation, or manuscript preparation.

\section{Availability of data and materials}

Data sharing is not applicable to this article as no datasets were generated or analyzed during the current study.

Ethics approval and consent to participate Not applicable.

\section{Consent for publication}

Not applicable.

\section{Competing interests}

The authors declare that they have no competing interests.

Received: 2 December 2019 Accepted: 9 March 2020

Published online: 04 May 2020

\section{References}

1. Canadian Institute for Health Information. Hospitalization disparities by socio-economic status for males and females. Ottawa: $\mathrm{ClH} ; 2010$.

2. Moy E, Chang E, Barrett M. Centers for disease C, prevention. Potentially preventable hospitalizations - United States, 2001-2009. MMWR Suppl. 2013: 62(3):139-43.

3. Tian Y, Dixon A, Gao H. Emergency hospital admissions for ambulatory caresensitive conditions: identifying the potential for reductions. London; 2012.

4. Weeks W, Ventelou B, Paraponaris A, Weeks WB. Rates of admission for ambulatory care sensitive conditions in France in 2009-2010: trends, geographic variation, costs, and an international comparison. Eur J Health Econ. 2016;17(4):453-70.

5. Dimitrovova K, Costa C, Santana P, Perelman J. Evolution and financial cost of socioeconomic inequalities in ambulatory care sensitive conditions: an ecological study for Portugal, 2000-2014. Int J Equity Health. 2017;16(1):145.

6. Page A, Ambrose S, Glover J, Hetzel D. Atlas of avoidable Hospitalisations in Australia: ambulatory care-sensitive conditions. Adelaide; 2007.

7. Purdy S, Griffin T, Salisbury C, Sharp D. Ambulatory care sensitive conditions: terminology and disease coding need to be more specific to aid policy makers and clinicians. Public Health. 2009;123(2):169-73.

8. Billings J, Zeitel L, Lukomnik J, Carey TS, Blank AE, Newman L. Impact of socioeconomic status on hospital use in New York City. Health Aff. 1993; 12(1):162-73.

9. Canadian Institute for Health Information. Ambulatory Care Sensitive Conditions Ottawa. ON: ClHI; 2018.

10. Falster $M$, Jorm L. A guide to the potentially preventable hospitalisations indicator in Australia. Sydney; 2017.

11. Canadian Institute for Health Information. Disparities in primary health care experiences among Canadians with ambulatory care sensitive conditions. Ottawa; 2012

12. Agabiti N, Pirani M, Schifano P, Cesaroni G, Davoli M, Bisanti L, et al. Income level and chronic ambulatory care sensitive conditions in adults: a multicity population-based study in Italy. BMC Public Health. 2009;9(1):457.

13. Sheringham J, Asaria M, Barratt $H$, Raine R, Cookson R. Are some areas more equal than others? Socioeconomic inequality in potentially avoidable emergency hospital admissions within English local authority areas. J Health Serv Res Policy. 2017;22(2):83-90. 
14. Löfqvist T, Burström B, Walander A, Ljung R. Inequalities in avoidable hospitalisation by area income and the role of individual characteristics: a population-based register study in Stockholm County, Sweden. BMJ Qual Saf. 2014:23(3):206-14

15. Payne RA, Abel GA, Guthrie B, Mercer SW. The effect of physical multimorbidity, mental health conditions and socioeconomic deprivation on unplanned admissions to hospital: a retrospective cohort study. CMAJ. 2013;185(5):E221-8.

16. Roos LL, Walld R, Uhanova J, Bond R. Physician visits, hospitalizations, and socioeconomic status: ambulatory care sensitive conditions in a Canadian setting. Health Serv Res. 2005:40(4):1167-85.

17. Doshi RP, Aseltine RH Jr, Sabina AB, Graham GN. Racial and ethnic disparities in preventable hospitalizations for chronic disease: prevalence and risk factors. J Racial Ethn Health Disparities. 2017;4(6):1100-6.

18. Dalla Zuanna T, Spadea T, Milana M, Petrelli A, Cacciani L, Simonato L, et al. Avoidable hospitalization among migrants and ethnic minority groups: a systematic review. Eur J Pub Health. 2017:27(5):861-8.

19. Rosano A, Loha CA, Falvo R, van der Zee J, Ricciardi W, Guasticchi G, et al. The relationship between avoidable hospitalization and accessibility to primary care: a systematic review. Eur J Pub Health. 2013;23(3):356-60.

20. van Loenen T, van den Berg MJ, Westert GP, Faber MJ. Organizational aspects of primary care related to avoidable hospitalization: a systematic review. Fam Pract. 2014;31(5):502-16.

21. Busby J, Purdy S, Hollingworth W. A systematic review of the magnitude and cause of geographic variation in unplanned hospital admission rates and length of stay for ambulatory care sensitive conditions. BMC Health Serv Res. 2015;15:324.

22. Ardura-Garcia C, Stolbrink M, Zaidi S, Cooper PJ, Blakey JD. Predictors of repeated acute hospital attendance for asthma in children: a systematic review and meta-analysis. Pediatr Pulmonol. 2018;53(9):1179-92.

23. Hawkins NM, Jhund PS, MCMurray JJ, Capewell S. Heart failure and socioeconomic status: accumulating evidence of inequality. Eur J Heart Fail. 2012;14(2):138-46

24. Gershon AS, Dolmage TE, Stephenson A, Jackson B. Chronic obstructive pulmonary disease and socioeconomic status: a systematic review. COPD. 2012;9(3):216-26.

25. Agency for Healthcare Research and Quality. Quality indicator user guide: Prevention Quality Indicators (PQI) composite measures. Rockville; 2019.

26. NHS Digital. Ambulatory Care Sensitive Conditions (ACSCs). Leeds: NHS Digital; 2019. Available from: https://digital.nhs.uk/data-and-information/ data-tools-and-services/data-services/innovative-uses-of-data/demand-onhealthcare/ambulatory-care-sensitive-conditions.

27. Allen L, Williams J, Townsend N, Mikkelsen B, Roberts N, Foster C, et al. Socioeconomic status and non-communicable disease behavioural risk factors in low-income and lower-middle-income countries: a systematic review. Lancet Glob Health. 2017;5(3):e277-e89.

28. Forrest LF, Adams J, Wareham H, Rubin G, White M. Socioeconomic inequalities in lung cancer treatment: systematic review and meta-analysis. PLoS Med. 2013;10(2):e1001376.

29. Houweling TA, Karim-Kos HE, Kulik MC, Stolk WA, Haagsma JA, Lenk EJ, et al. Socioeconomic inequalities in neglected tropical diseases: a systematic review. PLoS Negl Trop Dis. 2016;10(5):e0004546.

30. Marshall IJ, Wang Y, Crichton S, McKevitt C, Rudd AG, Wolfe CD. The effects of socioeconomic status on stroke risk and outcomes. Lancet Neurol. 2015; 14(12):1206-18.

31. Mustard CA, Etches J. Gender differences in socioeconomic inequality in mortality. J Epidemiol Community Health. 2003;57(12):974-80.

32. Probst C, Roerecke M, Behrendt S, Rehm J. Socioeconomic differences in alcohol-attributable mortality compared with all-cause mortality: a systematic review and meta-analysis. Int J Epidemiol. 2014;43(4):1314-27.

33. Rees I, Jones $D$, Chen $H$, Macleod U. Interventions to improve the uptake of cervical cancer screening among lower socioeconomic groups: a systematic review. Prev Med. 2018;111:323-35.

34. Scott A, Chambers D, Goyder E, O'Cathain A. Socioeconomic inequalities in mortality, morbidity and diabetes management for adults with type 1 diabetes: a systematic review. PLoS One. 2017;12(5):e0177210.

35. Sommer I, Griebler U, Mahlknecht P, Thaler K, Bouskill K, Gartlehner G, et al. Socioeconomic inequalities in non-communicable diseases and their risk factors: an overview of systematic reviews. BMC Public Health. 2015;15:914.

36. McCartney G, Vittal Katikireddi S, Walsh D, Arnot J, Leyland A. Trends in socio-economic inequalities in adult mortality in high and upper-middle income nations: a systematic review. PROSPERO: International prospective register of systematic reviews CRD42016025419; 2016.

37. Thomson K, Bambra C, McNamara C, Huijts T, Todd A. The effects of public health policies on population health and health inequalities in European welfare states: protocol for an umbrella review. Syst Rev. 2016;5:57.

38. Wang B, Zhan S, Gong T, Lee L. Iron therapy for improving psychomotor development and cognitive function in children under the age of three with iron deficiency anaemia. Cochrane Database Syst Rev. 2013;6: CD001444.

39. Salazar CA, Basilio Flores JE, Veramendi Espinoza LE, Mejia Dolores JW, Rey Rodriguez DE, Loza MC. Ranolazine for stable angina pectoris. Cochrane Database Syst Rev. 2017;2:CD011747.

40. Wang LW, Fahim MA, Hayen A, Mitchell RL, Baines L, Lord S, et al. Cardiac testing for coronary artery disease in potential kidney transplant recipients. Cochrane Database Syst Rev. 2011:12:CD008691.

41. Fisher SA, Doree C, Mathur A, Taggart DP, Martin-Rendon E. Stem cell therapy for chronic ischaemic heart disease and congestive heart failure. Cochrane Database Syst Rev. 2016;12:CD007888.

42. Huffman MD, Karmali KN, Berendsen MA, Andrei AC, Kruse J, McCarthy PM, et al. Concomitant atrial fibrillation surgery for people undergoing cardiac surgery. Cochrane Database Syst Rev 2016;8:CD011814.

43. Kramer S, Rottier BL, Scholten RJ, Boluyt N. Ciclesonide versus other inhaled corticosteroids for chronic asthma in children. Cochrane Database Syst Rev. 2013;2:CD010352.

44. Teo E, Lockhart K, Purchuri SN, Pushparajah J, Cripps AW, van Driel ML. Haemophilus influenzae oral vaccination for preventing acute exacerbations of chronic bronchitis and chronic obstructive pulmonary disease. Cochrane Database Syst Rev. 2017;6:CD010010.

45. McCarthy B, Casey D, Devane D, Murphy K, Murphy E, Lacasse Y. Pulmonary rehabilitation for chronic obstructive pulmonary disease. Cochrane Database Syst Rev. 2015;2:CD003793

46. Walters JA, Tang JN, Poole P, Wood-Baker R. Pneumococcal vaccines for preventing pneumonia in chronic obstructive pulmonary disease. Cochrane Database Syst Rev. 2017;1:CD001390.

47. Alabed S, Sabouni A, Al Dakhoul S, Bdaiwi Y, Frobel-Mercier AK. Betablockers for congestive heart failure in children. Cochrane Database Syst Rev. 2016;1:CD007037.

48. Maguire MJ, Jackson CF, Marson AG, Nolan SJ. Treatments for the prevention of sudden unexpected death in epilepsy (SUDEP). Cochrane Database Syst Rev. 2016;7:CD011792.

49. Mackay L, Kilbride L, Adamson KA, Chisholm J. Hormone replacement therapy for women with type 1 diabetes mellitus. Cochrane Database Syst Rev. 2013;6:CD008613.

50. El Dib R, Gameiro OL, Ogata MS, Modolo NS, Braz LG, Jorge EC, et al. Zinc supplementation for the prevention of type 2 diabetes mellitus in adults with insulin resistance. Cochrane Database Syst Rev. 2015;5:CD005525.

51. Tam TS, Wu MH, Masson SC, Tsang MP, Stabler SN, Kinkade A, et al. Eplerenone for hypertension. Cochrane Database Syst Rev. 2017;2: CD008996.

52. Cochrane Work. RCT filters for different databases. London: The Cochrane Collaboration; 2018. Available from: https://work.cochrane.org/rct-filtersdifferent-databases.

53. The World Bank. World Bank Country and Lending Groups. Washington, DC: The World Bank Group. 2018. Available from: https://datahelpdesk. worldbank.org/knowledgebase/articles/906519-world-bank-country-andlending-groups.

54. Joanna Briggs Institute. Critical Appraisal Tools North Adelaide, Australia: Joanna Briggs Institute; 2019. Available from: https://joannabriggs.org/ critical_appraisal_tools.

55. Asaria M, Ali S, Doran T, Ferguson B, Fleetcroft R, Goddard M, et al. How a universal health system reduces inequalities: lessons from England. J Epidemiol Community Health. 2016;70(7):637-43.

56. Aube-Maurice J, Rochette L, Blais C. Divergent associations between incident hypertension and deprivation based on different sources of case identification. Chronic Dis Inj Can. 2012;32(3):121-30

57. Bacon SL, Bouchard A, Loucks EB, Lavoie KL, Bacon SL, Bouchard A, et al. Individual-level socioeconomic status is associated with worse asthma morbidity in patients with asthma. Respir Res. 2009;10:125.

58. Banham D, Woollacott T, Gray J, Humphrys B, Mihnev A, McDermott R. Recognising potential for preventing hospitalisation. Aust Health Rev. 2010; 34(1):116-22. 
59. Begley CE, Basu R, Reynolds T, Lairson DR, Dubinsky S, Newmark M, et al. Sociodemographic disparities in epilepsy care: results from the Houston/ New York City health care use and outcomes study. Epilepsia. 2009;50(5): 1040-50.

60. Bocour A, Tria M. Preventable Hospitalization Rates and Neighborhood Poverty among New York City Residents, 2008-2013. J Urban Health. 2016 93(6):974-83.

61. Booth GL, Hux JE. Relationship between avoidable hospitalizations for diabetes mellitus and income level. Arch Intern Med. 2003;163(1):101-6.

62. Chen P-C, Tsai C-Y, Woung L-C, Lee Y-C. Socioeconomic disparities in preventable hospitalization among adults with diabetes in Taiwan: a multilevel modelling approach. Int J Equity Health. 2015;14:31.

63. Christensen S, Mogelvang R, Heitmann M, Prescott E. Level of education and risk of heart failure: a prospective cohort study with echocardiography evaluation. Eur Heart J. 2011;32(4):450-8

64. Davies S, Schultz E, Raven M, Wang NE, Stocks CL, Delgado MK, et al. Development and Validation of the Agency for Healthcare Research and Quality Measures of Potentially Preventable Emergency Department (ED) Visits: The ED Prevention Quality Indicators for General Health Conditions. Health Serv Res. 2017;52(5):1667-84.

65. Disano J, Goulet J, Muhajarine N, Neudorf C, Harvey J. Social-economic status and rates of hospital admission for chronic disease in urban Canada. Can Nurse. 2010;106(1):24-9.

66. Eisner MD, Blanc PD, Omachi TA, Yelin EH, Sidney S, Katz PP, et al. Socioeconomic status, race and COPD health outcomes. J Epidemiol Community Health. 2011;65(1):26-34.

67. Fleetcroft $R$, Asaria M, Ali S, Cookson R. Outcomes and inequalities in diabetes from 2004/2005 to 2011/2012: English longitudinal study. Br J Gen Pract. 2017;67(654):e1-9.

68. Govan L, Maietti E, Torsney B, Wu O, Briggs A, Colhoun HM, et al. The effect of deprivation and $\mathrm{HbA} 1 \mathrm{C}$ on admission to hospital for diabetic ketoacidosis in type 1 diabetes. Diabetologia. 2012;55(9):2356-60.

69. Gupta RP, Mukherjee M, Sheikh A, Strachan DP. Persistent variations in national asthma mortality, hospital admissions and prevalence by socioeconomic status and region in England. Thorax. 2018;73(8):706-12.

70. Jackson G, Tobias M. Potentially avoidable hospitalisations in New Zealand, 1989-98. Aust N Z J Public Health. 2001;25(3):212-21.

71. Lemstra M, Neudorf C, Opondo J. Health disparity by neighbourhood income. Can J Public Health. 2006;97(6):435-9.

72. Li X, Sundquist J, Sundquist K, Li X, Sundquist J, Sundquist K. Socioeconomic and occupational risk factors for epilepsy: a nationwide epidemiological study in Sweden. Seizure. 2008;17(3):254-60.

73. Macleod MR, Andrews PJD. Effect of deprivation and gender on the incidence and management of acute brain disorders. Intensive Care Med. 2002;28(12):1729-34

74. Prescott $E$, Lange $P$, Vestbo J. Socioeconomic status, lung function and admission to hospital for COPD: results from the Copenhagen City Heart Study. Eur Respir J. 1999;13(5):1109-14.

75. Quan H, Chen G, Tu K, Bartlett G, Butt DA, Campbell NRC, et al. Outcomes among 3.5 million newly diagnosed hypertensive Canadians. Can J Cardiol. 2013:29(5):592-7.

76. Roberts SE, Button LA, Hopkin JM, Goldacre MJ, Lyons RA, Rodgers SE, et al. Influence of social deprivation and air pollutants on serious asthma. Eur Respir J. 2012;40(3):785-8.

77. Shah RU, Winkleby MA, Van Horn L, Phillips LS, Eaton CB, Martin LW, et al. Education, income, and incident heart failure in post-menopausal women: The Women'S Health Initiative Hormone therapy Trials. J Am Coll Cardiol. 2011;58(14):1457-64

78. Shulman R, Luo J, Shah BR. Mental health visits and low socio-economic status in adolescence are associated with complications of Type 1 diabetes in early adulthood: a population-based cohort study. Diabet Med. 2018; 35(7):920-8

79. Walker RL, Chen G, McAlister FA, Campbell NRC, Hemmelgarn BR, Dixon E, et al. Hospitalization for uncomplicated hypertension: an ambulatory care sensitive condition. Can J Cardiol. 2013;29(11):1462-9.

80. Falster MO, Jorm LR, Douglas KA, Blyth FM, Elliott RF, Leyland AH. Sociodemographic and health characteristics, rather than primary care supply, are major drivers of geographic variation in preventable hospitalizations in Australia. Med Care. 2015;53(5):436-45.
81. Abel GA, Barclay ME, Payne RA. Adjusted indices of multiple deprivation to enable comparisons within and between constituent countries of the UK including an illustration using mortality rates. BMJ Open. 2016;6(11):e012750.

\section{Publisher's Note}

Springer Nature remains neutral with regard to jurisdictional claims in published maps and institutional affiliations.
Ready to submit your research? Choose BMC and benefit from:

- fast, convenient online submission

- thorough peer review by experienced researchers in your field

- rapid publication on acceptance

- support for research data, including large and complex data types

- gold Open Access which fosters wider collaboration and increased citations

- maximum visibility for your research: over $100 \mathrm{M}$ website views per year

At BMC, research is always in progress.

Learn more biomedcentral.com/submissions 